\title{
A Subordination Principle on Wright Functions and Regularized Resolvent Families
}

\author{
Luciano Abadias and Pedro J. Miana \\ Departamento de Matemáticas, Instituto Universitario de Matemáticas y Aplicaciones, Universidad de Zaragoza, \\ 50009 Zaragoza, Spain
}

Correspondence should be addressed to Pedro J. Miana; pjmiana@unizar.es

Received 11 December 2014; Accepted 20 February 2015

Academic Editor: Gestur Ólafsson

Copyright (c) 2015 L. Abadias and P. J. Miana. This is an open access article distributed under the Creative Commons Attribution License, which permits unrestricted use, distribution, and reproduction in any medium, provided the original work is properly cited.

\begin{abstract}
We obtain a vector-valued subordination principle for $\left(g_{\alpha}, g_{\beta}\right)$-regularized resolvent families which unified and improves various previous results in the literature. As a consequence, we establish new relations between solutions of different fractional Cauchy problems. To do that, we consider scaled Wright functions which are related to Mittag-Leffler functions, the fractional calculus, and stable Lévy processes. We study some interesting properties of these functions such as subordination (in the sense of Bochner), convolution properties, and their Laplace transforms. Finally we present some examples where we apply these results.
\end{abstract}

\section{Introduction}

A function $f:(0, \infty) \rightarrow \mathbb{R}$ is a Bernstein function if $f$ is of class $C^{\infty}, f(\lambda) \geq 0$ for all $\lambda>0$ and

$$
(-1)^{n-1} f^{(n)}(\lambda) \geq 0, \quad \lambda>0, n \in \mathbb{N} .
$$

The celebrated Bochner subordination theorem characterizes Bernstein functions: given $f$, a Bernstein function, there exists a unique convolution semigroup of subprobability measures $\left(\mu_{t}\right)_{t>0}$ on $[0, \infty)$ such that

$$
e^{-t f(\lambda)}=\int_{0}^{\infty} e^{-\lambda s} d \mu_{t}(s), \quad \mathfrak{R} \lambda>0 .
$$

Conversely, given a convolution semigroup of subprobability measures $\left(\mu_{t}\right)_{t>0}$ on $[0, \infty)$, then there exists a unique Bernstein function $f$ such that (2) holds true; see, for example, [1, Theorem 5.2]. The original subordination principle for stochastic processes in connection with diffusion equations and semigroups was introduced in [2]. In [3, Chapters 4.3, and 4.4], a detailed study of stochastic processes, their transition semigroups, generators, and subordination results are developed.

Now let $A$ be a densely defined closed linear operator on a Banach space $X$ which generates a $C_{0}$-contraction semigroup
$(T(t))_{t>0} \subset \mathscr{B}(X)$. Then the solution of the first order abstract Cauchy problem

$$
\begin{gathered}
u^{\prime}(t)=A u(t), \quad t>0, \\
u(0)=x
\end{gathered}
$$

is given by $u(t)=T(t) x$ for $t>0$. Now, suppose that $\left(\mu_{t}\right)_{t>0}$ is a vaguely continuous convolution semigroup of subprobability measures on $[0, \infty)$ with the corresponding Bernstein function $f$. Then the Bochner integral

$$
T^{f}(t) x:=\int_{0}^{\infty} T(s) x d \mu_{t}(s), \quad x \in X, t>0,
$$

defines again a $C_{0}$-contraction semigroup on $X[1$, Proposition 12.1]. Then the semigroup $\left(T^{f}(t)\right)_{t>0}$ is called subordinate (in line with Bochner) to the semigroup $(T(t))_{t>0}$ with respect to the Bernstein function $f$. In particular, given $0<\alpha<1$ and $d \mu_{t}(s)=f_{t, \alpha}(s) d s$ (where $f_{t, \alpha}$ are the stable Lévy processes, see (32)), then

$$
T^{(\alpha)}(t) x:=\int_{0}^{\infty} f_{t, \alpha}(s) T(s) x d s, \quad x \in X, t>0,
$$

is an analytic semigroup generated by $-(-A)^{\alpha}$, the fractional powers of the generator $A$ according to Balakrishnan. For more details, see [4, Chapter IX]. 
Other subordination formulae allow defining new families of operators from some previous ones by integration. Let $A$ be the generator of a cosine function $(C(t))_{t>0}$ on a Banach space $X$ (see definition in [5, Section 3.14]). Then $A$ generates a holomorphic $C_{0}$-semigroup $(T(z))_{z \in \mathbb{C}_{+}}$of angle $\pi / 2$, given by

$$
T(z) x=\frac{1}{\sqrt{\pi z}} \int_{0}^{\infty} e^{-s^{2} / 4 z} C(s) x d s, \quad x \in X, z \in \mathbb{C}_{+},
$$

[5, Theorem 3.14.17]. Remember that the solution of the second order Cauchy problem

$$
\begin{gathered}
u^{\prime \prime}(t)=A u(t), \quad t>0, \\
u(0)=x, \\
u^{\prime}(0)=0
\end{gathered}
$$

is $u(t)=C(t) x$ for $t>0$ [5, Section 3.14].

In [6], a two-kernel dependent family of strong continuous operators defined in a Banach space is introduced. This family allows us to consider in a unified treatment the notions of, among others, $C_{0}$-semigroups of operators, cosine families, $n$-times integrated semigroups, resolvent families, and $k$ generalized solutions. Let $a \in L_{\text {loc }}^{1}\left(\mathbb{R}_{+}\right)$and $k \in C\left(\mathbb{R}_{+}\right)$. The family $\left\{S_{a, k}(t)\right\}_{t>0} \subset \mathscr{B}(X)$ is a $(a, k)$-regularized resolvent family generated by $A$ if the following conditions are fulfilled: $S_{a, k}(t)$ is strongly continuous for $t>0$ and $S_{a, k}(0) x=k(0) x$ for all $x \in X ; S_{a, k}(t) A \subset A S_{a, k}(t)$, that is, $S_{a, k}(t) A(x) \subset$ $A S_{a, k}(t) x$, for $x \in D(A)$ and $t>0$; and

$$
\begin{array}{r}
S_{a, k}(t) x=k(t) x+A\left(\int_{0}^{t} a(t-s) S_{a, k}(s) x d s\right), \\
x \in X, \quad t>0 ;
\end{array}
$$

see [6, Definition 2.1]. In the case $k(t)=1$, we obtain the resolvent families which are treated in detail in [7]; for $k(t)=$ $a(t)=1$, this family of operators is a $C_{0}$-semigroup, and we also retrieve cosine functions for $k(t)=1$ and $a(t)=t(t>$ $0)$. Some spectral properties for $(a, k)$-regularized resolvent families are proved in [8].

Subordination theorems for $(a, k)$-regularized resolvents have been considered in some different works. In [7, Section I.4], the use of the theory of Bernstein functions, completely positive functions, and the Laplace transform allows showing some subordination results for $(a, 1)$-regularized resolvents [7, Theorem 4.1, Corollaries 4.4 and 4.5]. A nice subordination theorem for $m$-times integrated semigroup is proved in [6, Theorem 3.7]. In the case of $\left(t^{\alpha-1} / \Gamma(\alpha), 1\right)$-regularized functions, this subordination theorem is improved in [9, Theorem 3.1] and [10, Theorem 3.1] and an integral representation, similar to formula (6), is also proved involving Wright functions. In [11, Theorem 3.1], using holomorphic functional calculus, the authors prove a subordination result for $\left(t^{\alpha-1} / \Gamma(\alpha), 1\right)$-regularized resolvent families generated by fractional powers of closed operators, which extends both [4, Chapter IX, Section 11, Theorem 2] and [9, Theorem 3.1].
Finally, in [12, Theorem 2.8], a subordination principle for $(a, k)$-regularized resolvents, inspired in the original proof of [7, Theorem 4.1], is shown. In all these results, note that the subordination integral formula is only present in [9, Theorem 3.1] and [11, Theorem 3.1].

The main aim of this paper is to obtain subordination integral formulae to $\left(t^{\alpha-1} / \Gamma(\alpha), t^{\beta-1} / \Gamma(\beta)\right)$-regularized resolvents (Theorem 12). To achieve this, we present a detailed presentation of Wright and Mittag-Leffler functions in Section 2, which includes some basic results and known connections of these functions and fractional differential equations.

In Section 3, we introduce a new family of biparameter special functions $\psi_{\alpha, \beta}$ in two variables defined by scaling Wright functions (Definition 2). This family of functions $\psi_{\alpha, \beta}$ plays a fundamental role in the subordination principle for $\left(t^{\alpha-1} / \Gamma(\alpha), t^{\beta-1} / \Gamma(\beta)\right)$-regularized resolvent families; see formula (51). Moreover, these functions satisfy a nice subordination formula, Theorem 8 , which extends some known results for Wright M-function and stable Lévy processes; see Remark 9. In fact the algebraic nature (for convolution products) of these functions $\psi_{\alpha, \beta}$ is shown in Propositions 5 and 7.

In Section 4 , we consider $\left(t^{\alpha-1} / \Gamma(\alpha), t^{\beta-1} / \Gamma(\beta)\right)$-regularized resolvents in abstract Banach spaces. We prove the main subordination principle, Theorem 12, and some consequences in Remark 13 and Corollary 14.

Finally, in Section 5 we present some comments, concrete examples, and applications to fractional Cauchy problems which illustrate the main results of this paper.

Notation. Let $\mathbb{R}_{+}:=[0, \infty), \mathbb{C}_{+}:=\{z \in \mathbb{C}: \mathfrak{R} z>0\}$, and $L^{1}\left(\mathbb{R}_{+}\right)$be the Lebesgue Banach algebra of integrable function on $\mathbb{R}_{+}$with the usual convolution product

$$
f * g(t)=\int_{0}^{t} f(t-s) g(s) d s, \quad f, g \in L^{1}\left(\mathbb{R}_{+}\right), t \geq 0 .
$$

The usual Laplace transform of a function $f, \widehat{f}$, is defined by

$$
\widehat{f}(\lambda)=\int_{0}^{\infty} f(t) e^{-\lambda t} d t, \quad \lambda \in \mathbb{C}_{+},
$$

for $f \in L^{1}\left(\mathbb{R}_{+}\right)$. Let $\gamma>0$, and we denote $g_{\gamma}(t):=t^{\gamma-1} / \Gamma(\gamma)$, $t>0$, and $\widehat{g_{\gamma}}(\lambda)=1 / \lambda^{\gamma}$ for $\lambda \in \mathbb{C}_{+}$.

The set of continuous functions on $\mathbb{R}_{+}$such that $\lim _{t \rightarrow \infty}|f(t)|=0$ is denoted as $C_{0}\left(\mathbb{R}_{+}\right)$, and the set of holomorphic functions on $\mathbb{C}_{+}$such that $\lim _{|z| \rightarrow \infty}|f(z)|=0$ is denoted as $\mathscr{H}_{0}\left(\mathbb{C}^{+}\right)$. We denote by $X$ an abstract Banach space, $\mathscr{B}(X)$ the set of linear and bounded operators on the Banach space $X$, and $C_{c}^{(\infty)}\left(\mathbb{R}_{+} ; X\right)$ the set of functions of compact support and infinitely differentiable on $\mathbb{R}_{+}$into $X$.

\section{Mittag-Leffler and Wright Functions}

In this section we present definitions and basic properties of Mittag-Leffler and Wright functions. The algebraic structure of those functions has been partially considered in [13] and formulae (15) and (16) seem to be new. 
The Mittag-Leffler functions are defined by

$$
E_{\alpha, \beta}(z):=\sum_{n=0}^{\infty} \frac{z^{n}}{\Gamma(\alpha n+\beta)}, \quad \alpha, \beta>0, z \in \mathbb{C} .
$$

We write $E_{\alpha}(z):=E_{\alpha, 1}(z)$. The Mittag-Leffler functions satisfy the following fractional differential problems:

$$
\begin{aligned}
& { }_{C} D_{t}^{\alpha} E_{\alpha}\left(\omega t^{\alpha}\right)=\omega E_{\alpha}\left(\omega t^{\alpha}\right), \\
& { }_{R} D_{t}^{\alpha}\left(t^{\alpha-1} E_{\alpha, \alpha}\left(\omega t^{\alpha}\right)\right)=\omega t^{\alpha-1} E_{\alpha, \alpha}\left(\omega t^{\alpha}\right) \text {, }
\end{aligned}
$$

for $0<\alpha<1$, under certain initial conditions, where ${ }_{C} D_{t}^{\alpha}$ and ${ }_{R} D_{t}^{\alpha}$ denote the Caputo and Riemann-Liouville fractional derivatives of order $\alpha$, respectively; see Section 5 and $[14,15]$. Their Laplace transform is

$$
\int_{0}^{\infty} e^{-\lambda t} t^{\beta-1} E_{\alpha, \beta}\left(\omega t^{\alpha}\right) d t=\frac{\lambda^{\alpha-\beta}}{\lambda^{\alpha}-\omega}, \quad \Re \lambda>\omega^{1 / \alpha}, \omega>0 .
$$

For more details see [10, Section 1.3].

Recently, the next algebraic property has been proved

$$
\begin{aligned}
& \left(\int_{t}^{t+s}-\int_{0}^{s}\right) \frac{E_{\alpha}\left(\omega r^{\alpha}\right)}{(t+s-r)^{\alpha}} d r \\
& \quad=\alpha \int_{0}^{t} \int_{0}^{s} \frac{E_{\alpha}\left(\omega r_{1}^{\alpha}\right) E_{\alpha}\left(\omega r_{2}^{\alpha}\right)}{\left(t+s-r_{1}-r_{2}\right)^{1+\alpha}} d r_{1} d r_{2}, \quad t, s \geq 0,
\end{aligned}
$$

for $0<\alpha<1$ and $\omega \in \mathbb{C}$; see [13, Theorem 1]. In fact, a similar identity holds for generalized Mittag-Leffler function $E_{\alpha, \beta}$ with $0<\alpha<1, \beta>\alpha$, and

$$
\begin{aligned}
& \left(\int_{t}^{t+s}-\int_{0}^{s}\right) \frac{(t+s-r)^{\beta-\alpha-1}}{\Gamma(\beta-\alpha)} r^{\beta-1} E_{\alpha, \beta}\left(\omega r^{\alpha}\right) d r \\
& \quad=\frac{\alpha}{\Gamma(1-\alpha)} \int_{0}^{t} \int_{0}^{s} \frac{r_{1}^{\beta-1} E_{\alpha, \beta}\left(\omega r_{1}^{\alpha}\right) r_{2}^{\beta-1} E_{\alpha, \beta}\left(\omega r_{2}^{\alpha}\right)}{\left(t+s-r_{1}-r_{2}\right)^{1+\alpha}} d r_{1} d r_{2},
\end{aligned}
$$

for $t, s \geq 0$ and $\omega>0$. The proof of this result is a straightforward consequence of [16, Theorem 5]. In the case $\beta=\alpha$ for $0<\alpha<1$, the algebraic property is

$$
\begin{aligned}
& (t+s)^{\alpha-1} E_{\alpha, \alpha}\left(\omega(t+s)^{\alpha}\right) \\
& =\frac{\alpha}{\Gamma(1-\alpha)} \int_{0}^{t} \int_{0}^{s} \frac{r_{1}^{\alpha-1} E_{\alpha, \alpha}\left(\omega r_{1}^{\alpha}\right) r_{2}^{\alpha-1} E_{\alpha, \alpha}\left(\omega r_{2}^{\alpha}\right)}{\left(t+s-r_{1}-r_{2}\right)^{1+\alpha}} d r_{1} d r_{2},
\end{aligned}
$$

which is a direct consequence of Theorems 2.1 and 2.2 of [17].

The Wright function that we denote by $W_{\lambda, \mu}$ was introduced and investigated by $E$. Maitland Wright in a series of notes starting from 1933 in the framework of the theory of partitions; see [18]. This entire function is defined by the series representation, convergent in the whole complex plane:

$$
W_{\lambda, \mu}(z):=\sum_{n=0}^{\infty} \frac{z^{n}}{n ! \Gamma(\lambda n+\mu)}, \quad \lambda>-1, \mu \in \mathbb{C} .
$$

The equivalence between the above series and the following integral representations of $W_{\lambda, \mu}$ is easily proven by using the Hankel formula for the Gamma function:

$$
W_{\lambda, \mu}(z)=\frac{1}{2 \pi i} \int_{\mathrm{Ha}} \sigma^{-\mu} e^{\sigma+z \sigma^{-\lambda}} d \sigma, \quad \lambda>-1, \mu \geq 0, z \in \mathbb{C},
$$

where Ha denotes the Hankel path defined as a contour that begins at $t=-\infty-i a(a>0)$, encircles the branch cut that lies along the negative real axis, and ends up at $t=-\infty+i b(b>$ 0 ); for more details see [14, Appendix F]. It is clear that

$$
\frac{d}{d z} W_{\lambda, \mu}(z)=W_{\lambda, \lambda+\mu}(z), \quad z \in \mathbb{C}
$$

In addition, as discussed below, the following special cases are of considerable interest:

$$
\begin{array}{r}
M_{\alpha}(z):=W_{-\alpha, 1-\alpha}(-z), \quad F_{\alpha}(z):=W_{-\alpha, 0}(-z), \\
0<\alpha<1, \quad z \in \mathbb{C},
\end{array}
$$

interrelated through

$$
F_{\alpha}(z)=\alpha z M_{\alpha}(z), \quad z \in \mathbb{C} .
$$

The Wright $\mathbb{M}$-function in two variables $\mathbb{M}$ is defined by

$$
\mathbb{M}_{\alpha}(s, t):=t^{-\alpha} M_{\alpha}\left(s t^{-\alpha}\right), \quad t>0, s \in \mathbb{R} .
$$

This function has been studied, for example, in [14, page 257] and [19, Section 6]; a subordination formula for time fractional diffusion process is given in [19, Formula (6.3)] and [14, Formula (F.55)]: for $\eta, \beta \in(0,1)$, the following subordination formula holds true;

$$
\mathbb{M}_{\eta \beta}(s, t)=\int_{0}^{\infty} \mathbb{M}_{\eta}(s, \tau) \mathbb{M}_{\beta}(\tau, t) d \tau, \quad t, s>0 .
$$

This subordination formula had previously appeared in [10, Formula (3.28)].

The deep connection between fractional differential equations (in space and in time) and Wright-type functions $\left(W_{\lambda, \mu}, M_{\alpha}, F_{\alpha}, \mathbb{M}_{\alpha}, \ldots\right)$ has been studied in detail in $[14,19$, 20].

It is known that

$$
\begin{array}{r}
E_{\alpha, \alpha+\beta}(z)=\int_{0}^{\infty} e^{z t} W_{-\alpha, \beta}(-t) d t, \\
z \in \mathbb{C}, \quad 0<\alpha<1, \quad \beta \geq 0 ;
\end{array}
$$

that is, $E_{\alpha, \alpha+\beta}(-(\cdot))$ is the Laplace transform of $W_{-\alpha, \beta}(-(\cdot))$ in the whole complex plane; see [14, Formula (F.25)]. Then, observe that for $0<\alpha<1$

$$
\begin{array}{r}
E_{\alpha}(z)=\int_{0}^{\infty} e^{z t} M_{\alpha}(t) d t, \\
E_{\alpha, \alpha}(z)=\int_{0}^{\infty} e^{z t} F_{\alpha}(t) d t, \\
z \in \mathbb{C},
\end{array}
$$


where both functions are related to the solutions of the fractional differential problems mentioned above.

Nice connections between Mittag-Leffler functions and Wright functions are obtained by the Laplace transform; see formula (24) and

$$
\int_{0}^{\infty} e^{-z t} W_{\lambda, \mu}( \pm r) d r=\frac{1}{z} E_{\lambda, \mu}\left( \pm \frac{1}{z}\right), \quad|z|>0, \lambda>0
$$

[14, Formula (F.22)]. In the next proposition, we present some interesting properties of Wright functions. The next result extends the study which was done in [10, Chapter 1, page 14] for the case $W_{-\alpha, 1-\alpha}$ with $0<\alpha<1$.

Proposition 1. Let $0<\alpha<1$ and $\beta \geq 0$. Then the following properties hold:

(i) $\int_{0}^{\infty}\left(t^{\eta-1} / \Gamma(\eta)\right) W_{-\alpha, \beta}(-t) d t=1 / \Gamma(\alpha \eta+\beta) ; \eta>0$.

(ii) $W_{-\alpha, \beta}(-t) \geq 0$, for $t>0$.

Proof. (i) Using the definition of $W_{-\alpha, \beta}$, we have

$$
\begin{aligned}
& \int_{0}^{\infty} \frac{t^{\eta-1}}{\Gamma(\eta)} W_{-\alpha, \beta}(-t) d t \\
& \quad=\frac{1}{2 \pi i} \int_{\mathrm{Ha}} e^{\sigma} \sigma^{-\beta-\alpha \eta} d \sigma=g_{\beta+\alpha \eta}(1),
\end{aligned}
$$

where we have applied the Fubini theorem and the Laplace transform of $g_{\eta}$.

(ii) The positivity of $W_{-\alpha, \beta}(-t)$ follows from (24), the complete monotonicity of $E_{\alpha, \gamma}(-t)$ for $t>0,0<\alpha<1$, and $\gamma \geq \alpha$ (see [14, Appendix E, Formula (E.32)]), and the PostWidder inversion formula; see [10, Lemma 1.6].

\section{Scaled Wright Functions}

In this section, we introduce two-parameter Wright functions in Definition 2, which we call scaled Wright functions. This class of functions includes the Wright $\mathbb{M}$-function introduced in [19, Formula (6.2)] and also considered in [14, Formula (F.51)] and stable Lévy processes. They satisfy important properties (Theorem 3 and Proposition 5) and a subordination principle (Theorem 8 ) and play a crucial role in this paper.

Definition 2. For $0<\alpha<1$ and $\beta \geq 0$, we define the function $\psi_{\alpha, \beta}$ in two variables by

$$
\psi_{\alpha, \beta}(t, s):=t^{\beta-1} W_{-\alpha, \beta}\left(-s t^{-\alpha}\right), \quad t>0, s \in \mathbb{C} .
$$

Note that, using the change of variable $z=\sigma / t$, we get the integral representation

$$
\psi_{\alpha, \beta}(t, s)=\frac{1}{2 \pi i} \int_{\mathrm{Ha}} z^{-\beta} e^{t z-s z^{\alpha}} d z, \quad t, s>0 .
$$

The function $\psi_{\alpha, \beta}$ is considered in the literature in some particular cases. (i) For $\beta=1-\alpha$,

$$
\psi_{\alpha, 1-\alpha}(t, s)=t^{-\alpha} M_{\alpha}\left(s t^{-\alpha}\right)=\mathbb{M}_{\alpha}(s, t)=\varphi_{t, \alpha}(s), \quad t, s>0,
$$

where $\mathbb{M}_{\alpha}(s, t)$ is the Wright $\mathbb{M}$-function in two variables studied in [14, page 257] and $\varphi_{t, \alpha}(s)$ is considered in [10, Theorem 3.1]; for $\alpha=1 / 2$,

$$
\psi_{1 / 2,1 / 2}(t, s)=\frac{1}{\sqrt{\pi t}} e^{-s^{2} / 4 t}, \quad t, s>0 ;
$$

see [14, Appendix F, Formula (F.16)].

(ii) $\operatorname{For} \beta=0$,

$$
\psi_{\alpha, 0}(t, s)=\frac{1}{2 \pi i} \int_{\mathrm{Ha}} e^{t z-s z^{\alpha}} d z=: f_{s, \alpha}(t), \quad t, s>0,
$$

is the stable Lévy process of order $\alpha$; see Section 1, [2], and [4, Chapter IX], in particular

$$
\psi_{1 / 2,0}(t, s)=\frac{1}{2 \sqrt{\pi}} t^{-3 / 2} s e^{-s^{2} / 4 t}, \quad t, s>0
$$

In the next theorem, we join some properties which are satisfied by functions $\psi_{\alpha, \beta}$.

Theorem 3. Let $0<\alpha<1$ and $\beta \geq 0$; one has the following.

(i) $\psi_{\alpha, \beta}(t, s) \geq 0$, for $t, s>0$.

(ii) $\int_{0}^{\infty} e^{-\lambda t} \psi_{\alpha, \beta}(t, s) d t=\lambda^{-\beta} e^{-\lambda^{\alpha} s}$, for $s, \lambda>0$.

(iii) $\int_{0}^{\infty} e^{\lambda s} \psi_{\alpha, \beta}(t, s) d s=t^{\alpha+\beta-1} E_{\alpha, \alpha+\beta}\left(\lambda t^{\alpha}\right)$, for $t>0, \lambda \in$

(iv) $\iint_{0}^{\infty} e^{-\lambda s} e^{-\mu t} \psi_{\alpha, \beta}(t, s) d s d t=1 / \mu^{\beta}\left(\mu^{\alpha}+\lambda\right)$ for $t>0$, $\lambda, \mu>0$.

(v) $\psi_{\alpha, \beta+\gamma}(t, s)=\left(g_{\gamma} * \psi_{\alpha, \beta}(\cdot, s)\right)(t)$, for $t, s, \gamma>0$.

(vi) $\int_{0}^{\infty} g_{\eta}(s) \psi_{\alpha, \beta}(t, s) d s=g_{\alpha \eta+\beta}(t)$, for $t, \eta>0$.

Proof. (i) It is clear by Definition 2 and Proposition 1. (ii) It is easy to see that $\psi_{\alpha, \beta}(t, s)$ is of exponential growth in $t$. Let $\lambda>$ $\max (a, b)>0$, (where $a$ and $b$ are involved in the definition of Hankel path Ha). Then, by Cauchy theorem of residue,

$$
\int_{0}^{\infty} e^{-\lambda t} \psi_{\alpha, \beta}(t, s) d t=\frac{1}{2 \pi i} \int_{\mathrm{Ha}} \frac{z^{-\beta} e^{-s z^{\alpha}}}{z-\lambda} d z=\lambda^{-\beta} e^{-s \lambda^{\alpha}}
$$

(iii) Using (24), the result is direct by a change of variable. (iv) We combine parts (ii) and (iii) to obtain the equality. (v) It is clear using Laplace transform and (i). (vi) It is clear by a change of variable and applying Proposition 1(i).

We combine Theorem 3(ii) and (iii) and formula (13) to get the following corollary.

Corollary 4. For $0<\alpha, \gamma<1$, one denotes by $\Psi_{\gamma, \alpha}$ the function given by

$$
\Psi_{\gamma, \alpha}(t, s):=\int_{0}^{\infty} \psi_{\gamma, 0}(t, u) \psi_{\alpha, 0}(s, u) d u, \quad t, s>0 .
$$


Then $\Psi_{\gamma, \alpha}(t, s)=\Psi_{\alpha, \gamma}(s, t)$, and

$$
\begin{gathered}
\int_{0}^{\infty} e^{-\lambda t} \Psi_{\gamma, \alpha}(t, s) d t=s^{\alpha-1} E_{\alpha, \alpha}\left(-\lambda^{\gamma} s^{\alpha}\right), \quad s>0, \\
\iint_{0}^{\infty} e^{-\mu s} e^{-\lambda t} \Psi_{\gamma, \alpha}(t, s) d t d s=\frac{1}{\lambda^{\gamma}+\mu^{\alpha}}
\end{gathered}
$$

for $\mu, \lambda>0$.

Note that

$$
\Psi_{1 / 2,1 / 2}(t, s)=\frac{1}{2 \sqrt{\pi}(t+s)^{3 / 2}}, \quad t, s>0 .
$$

The following key lemma includes the particular case $\alpha=$ $\beta=1 / 2$ and $0<\eta$ proved in [21, Lemma 1$]$.

Proposition 5. For $0<\alpha<1, \beta \geq 0$, and $0<\eta$, the following identity holds:

$$
\psi_{\alpha, \beta+\alpha \eta}(t, u)=\int_{u}^{\infty} g_{\eta}(s-u) \psi_{\alpha, \beta}(t, s) d s
$$

for $t, u>0$.

Proof. Note that $\psi_{\alpha, \beta}$ is a Laplace transformable function and locally integrable in two variables. We apply the Laplace transform in variable $t$ to get in the right side

$$
\begin{aligned}
\int_{0}^{\infty} & e^{-\lambda t} \int_{u}^{\infty} g_{\eta}(s-u) \psi_{\alpha, \beta}(t, s) d s d t \\
= & \lambda^{-\beta} \int_{u}^{\infty} g_{\eta}(s-u) e^{-\lambda^{\alpha} s} d s=\lambda^{-\beta} \lambda^{-\alpha \eta} e^{-\lambda^{\alpha} u}
\end{aligned}
$$

with $\lambda>0$, where we have applied Theorem 3(ii) and [15, Chapter II, Formula (5.11)].

In the left side, we also apply the Laplace transform in the variable $t$ to get that

$$
\int_{0}^{\infty} e^{-\lambda t} \psi_{\alpha, \beta+\alpha \eta}(t, u) d t=\lambda^{-(\beta+\alpha \eta)} e^{-\lambda^{\alpha} u}, \quad u>0
$$

with $\lambda>0$, where we have used Theorem 3(ii).

Remark 6. For $u=0$ in Proposition 5, we obtain the equality

$$
\psi_{\alpha, \beta+\alpha \eta}(t, 0)=\int_{0}^{\infty} g_{\eta}(s) \psi_{\alpha, \beta}(t, s) d s=g_{\alpha \eta+\beta}(t), \quad t>0,
$$

proved in Theorem 3(vi).

Finally, we show an algebraic identity which satisfies functions $\psi_{\alpha, 1-\alpha}$.

Proposition 7. Take $0<\alpha<1, \beta>\alpha$, and $t, s>0$. Then

(i) $\left(\int_{t}^{t+s}-\int_{0}^{s}\right)\left((t+s-r)^{\beta-\alpha-1} / \Gamma(\beta-\alpha)\right) \psi_{\alpha, \beta-\alpha}(r, u) d r=$ $(\alpha / \Gamma(1-\alpha)) \int_{0}^{t} \int_{0}^{s}\left(\left(\psi_{\alpha, \beta-\alpha}\left(r_{1},(\cdot)\right) * \psi_{\alpha, \beta-\alpha}\left(r_{2},(\cdot)\right)\right)(u) /\right.$ $\left.\left(t+s-r_{1}-r_{2}\right)^{1+\alpha}\right) d r_{1} d r_{2}$; (ii) $\psi_{\alpha, 0}(t+s, u)=(\alpha / \Gamma(1-\alpha)) \int_{0}^{t} \int_{0}^{s}\left(\left(\psi_{\alpha, 0}\left(r_{1},(\cdot)\right) *\right.\right.$ $\left.\left.\psi_{\alpha, 0}\left(r_{2},(\cdot)\right)\right)(u) /\left(t+s-r_{1}-r_{2}\right)^{1+\alpha}\right) d r_{1} d r_{2}$.

Proof. Note that $\widehat{\psi \alpha, \beta-\alpha}(r,(\cdot))(\lambda)=r^{\beta-1} E_{\alpha, \beta}\left(-\lambda r^{\alpha}\right)$, for $\beta \geq$ $\alpha, r>0$, and $\lambda \in \mathbb{C}$; see Theorem 3(iii). Then we apply the Laplace transform in the variable $u$ in both equalities to get identities (15) and (16). The injectivity of Laplace transform allows us to finish the proof.

To finish this section, we prove a subordination formula for functions $\psi_{\alpha, \beta}$ which expands some well-known results.

Theorem 8. For $0<\alpha, \delta<1, \beta \geq \alpha$, and $\delta \geq \gamma$, the following identity holds:

$$
\begin{aligned}
& \psi_{\alpha \gamma, \beta-\alpha+\alpha(\delta-\gamma)}(t, s) \\
& \quad=\int_{0}^{\infty} \psi_{\alpha, \beta-\alpha}(t, r) \psi_{\gamma, \delta-\gamma}(r, s) d r, \quad t, s>0 .
\end{aligned}
$$

Proof. To show this theorem, we apply the Laplace transform in both variables $(t, s)$, the so-called double Laplace transform, Fubini theorem, Theorem 3(ii) and (iii), and finally formula (13) to get that

$$
\begin{aligned}
& \iint_{0}^{\infty} e^{-\lambda t-\mu s} \int_{0}^{\infty} \psi_{\alpha, \beta-\alpha}(t, r) \psi_{\gamma, \delta-\gamma}(r, s) d r d s d t \\
& \quad=\int_{0}^{\infty} \lambda^{\alpha-\beta} e^{-\lambda^{\alpha} r} r^{\gamma-1} E_{\gamma, \delta}\left(-\mu r^{\gamma}\right) d r \\
& =\frac{\lambda^{\alpha \gamma-(\beta-\alpha+\alpha \gamma)}}{\lambda^{\alpha \gamma}+\mu},
\end{aligned}
$$

for $\mathfrak{R} \lambda, \mathfrak{R} \mu>0$. Due to Theorem 3(iv) and the uniqueness of the double Laplace transform (see, e.g., [22, page 346]), we conclude the equality.

Remark 9. In the case that $\beta=\delta=1$, we obtain formula (23). For $\alpha=\beta$ and $\gamma=\delta$, we get the following subordination formula for stable Lévy processes:

$$
f_{s, \alpha \gamma}(t)=\int_{0}^{\infty} f_{r, \alpha}(t) f_{s, \gamma}(r) d r, \quad s, t>0,
$$

for $0<\alpha, \gamma<1$. Finally, for $\alpha=\gamma=1 / 2$ and $\beta=\delta=1$, we obtain that

$$
\psi_{1 / 4,3 / 4}(t, s)=\frac{1}{\pi \sqrt{t}} \int_{0}^{\infty} e^{-r^{2} / 4 t-s^{2} / 4 r} \frac{d r}{\sqrt{r}}, \quad t, s>0,
$$

where we use equality (31).

\section{Subordination Principle for Regularized Resolvent Families}

In the following we consider that the operator $A$ is a densely defined closed linear operator on a Banach space $X$. Let $\alpha, \beta>$ 0 . A family $\left\{S_{\alpha, \beta}(t)\right\}_{t>0} \subset \mathscr{B}(X)$ is a $\left(g_{\alpha}, g_{\beta}\right)$-regularized resolvent family generated by $A$ if the following conditions are satisfied. 
(a) $S_{\alpha, \beta}(t)$ is strongly continuous for $t>0$ and $\lim _{t \rightarrow 0^{+}} S_{\alpha, \beta}(t) x / g_{\beta}(t)=x$ for all $x \in X$.

(b) $S_{\alpha, \beta}(t) A \subset A S_{\alpha, \beta}(t)$; that is, $S_{\alpha, \beta}(t) A(x) \subset A S_{\alpha, \beta}(t) x$ for $x \in D(A)$ and $t>0$.

(c) The integral equation

$$
S_{\alpha, \beta}(t) x=\frac{t^{\beta-1}}{\Gamma(\beta)} x+A\left(\int_{0}^{t} \frac{(t-s)^{\alpha-1}}{\Gamma(\alpha)} S_{\alpha, \beta}(s) x d s\right)
$$

holds for $x \in X$ and $t>0$.

This family of operators was formerly introduced for general kernels $(a, k)$ in [6, Definition 2.1]. The above definition is also considered for $\alpha>0$ and $\beta \geq 1$ in [23], for $0<\alpha=\beta<1$ in [17], and for $\alpha>0$ and $\beta=1$ in [9, Definition 2.1].

The reason why we do not consider the value of $S_{\alpha, \beta}(\cdot)$ at 0 in condition (a) (compare with [6, Definition 2.1, Condition (R1)]) is that the function $t \mapsto g_{\beta}(t)$ has a singularity at 0 if $0<\beta<1$.

Let $S:(0, \infty) \rightarrow \mathscr{B}(X)$ be a strongly continuous operator family such that $S(\cdot) x \in L_{\text {loc }}^{1}\left(\mathbb{R}_{+}, X\right)$, for any $x \in X$. The operator family $\{S(t)\}_{t>0}$ is said to be Laplace-transformable if there exists $\omega \in \mathbb{R}$ such that the Laplace transform of $S$,

$$
\widehat{S}(\lambda) x=\int_{0}^{\infty} e^{-\lambda t} S(t) x d t, \quad \Re \lambda>\omega
$$

converges for $x \in X$; see, for example, [5, Definition 3.1.4]. If $A$ generates a $\left(g_{\alpha}, g_{\beta}\right)$-regularized resolvent family $\left\{S_{\alpha, \beta}(t)\right\}_{t>0}$ such that $S(\cdot) x \in L_{\text {loc }}^{1}\left(\mathbb{R}_{+}, X\right)$ for $x \in X$ and is Laplace transformable of parameter $\omega$, we write $A \in$ $\mathscr{C}^{\alpha, \beta}(\omega)$. We denote $\mathscr{C}^{\alpha, \beta}:=\bigcup\left\{\mathscr{C}^{\alpha, \beta}(\omega) ; \omega \geq 0\right\}$. The case of $\left(g_{\alpha}, 1\right)$-regularized resolvent families exponentially bounded, $\left\|S_{\alpha, 1}(t)\right\| \leq M e^{w t}$ for $t>0$, is considered in [11, Definition 2.5] and [10, Definition 2.4]; in this case, $\mathscr{C}^{\alpha}(\omega):=\mathscr{C}^{\alpha, 1}(\omega)$.

The next theorem characterizes the Laplace transform of $\left(g_{\alpha}, g_{\beta}\right)$-regularized family and extends [23, Theorem 3.11] and the proof is similar to the proof of [6, Proposition 3.1].

Theorem 10. Let $\alpha, \beta>0$. Then $A \in \mathscr{C}^{\alpha, \beta}(\omega)$ if and only if $\left(\omega^{\alpha}, \infty\right) \subset \rho(A)$ and there exists a strongly continuous function $S_{\alpha, \beta}(\cdot):(0, \infty) \rightarrow \mathscr{B}(X)$, locally integrable, $\lim _{t \rightarrow 0^{+}}\left(S_{\alpha, \beta}(t) x / g_{\beta}(t)\right)=x$ for all $x \in X$, and Laplace transformable such that

$$
\int_{0}^{\infty} e^{-\lambda t} S_{\alpha, \beta}(t) x d t=\lambda^{\alpha-\beta}\left(\lambda^{\alpha}-A\right)^{-1} x, \quad \lambda>\omega,
$$

for all $x \in X$. Furthermore, the family $\left\{S_{\alpha, \beta}(t)\right\}_{t>0}$ is the $\left(g_{\alpha}, g_{\beta}\right)$-regularized resolvent family generated by $A$.

Example 11. For $0<\alpha<1$ and $\beta \geq \alpha$, the family $\left\{S_{\alpha, \beta}(t)\right\}_{t>0}$ defined by

$$
\begin{aligned}
S_{\alpha, \beta}(t) f(s) & :=\left(\psi_{\alpha, \beta-\alpha}(t,(\cdot)) * f\right)(s) \\
= & \int_{0}^{s} f(s-u) \psi_{\alpha, \beta-\alpha}(t, u) d u, \\
& s \in \mathbb{R}_{+}, \quad f \in L^{1}\left(\mathbb{R}_{+}\right),
\end{aligned}
$$

is a $\left(g_{\alpha}, g_{\beta}\right)$-regularized resolvent family generated by $A=$ $-d / d t$ on the Banach space $L^{1}\left(\mathbb{R}_{+}\right)$. First note that the family is well defined since $\left\|\psi_{\alpha, \beta-\alpha}(t,(\cdot))\right\|_{1}=g_{\beta}(t)$ for $t>0$; see Theorem 3(vi). In addition, by Theorem 3(ii), we have that

$$
\begin{aligned}
\int_{0}^{\infty} & e^{-\lambda t} S_{\alpha, \beta}(t) f(s) d t \\
& =\int_{0}^{s} f(s-u) \int_{0}^{\infty} e^{-\lambda t} \psi_{\alpha, \beta-\alpha}(t, u) d t d u \\
& =\lambda^{\alpha-\beta} \int_{0}^{s} e^{-\lambda^{\alpha} u} f(s-u) d u \\
& =\lambda^{\alpha-\beta}\left(\lambda^{\alpha}+\frac{d}{d t}\right)^{-1} f(s), \quad s \in \mathbb{R}_{+} .
\end{aligned}
$$

The strong continuity at the origin is a simple check, and the reader can follow the steps on the proof of Theorem 12; in particular, the stable Lévy processes $\left(f_{(\cdot), \alpha}(t)\right)_{t>0}$ are convolution $\left(g_{\alpha}, g_{\alpha}\right)$-regularized resolvent families. Similarly, for $\lambda \epsilon$ $\mathbb{C}$, Mittag-Leffler functions $\left(t^{\beta-1} E_{\alpha, \beta}\left(-\lambda t^{\alpha}\right)\right)_{t \geq 0}$ are $\left(g_{\alpha}, g_{\beta}\right)$ regularized resolvent families generated by $A f(z)=-\lambda f(z)$ on the Banach space $C_{0}\left(\mathbb{R}_{+}\right)$(or $\mathscr{H}_{0}\left(\mathbb{C}_{+}\right)$); see formula (13). These two families of functions are canonical examples of $\left(g_{\alpha}, g_{\beta}\right)$-regularized resolvent families.

The next theorem is the main one of this paper.

Theorem 12. Let $0<\eta_{1} \leq 2,0<\eta_{2}$, and $\omega \geq 0$. If $A \in$ $\mathscr{C}^{\eta_{1}, \eta_{2}}(\omega)$ generates a $\left(g_{\eta_{1}}, g_{\eta_{2}}\right)$-regularized resolvent family $\left\{S_{\eta_{1}, \eta_{2}}(t)\right\}_{t>0}$, then $A \in \mathscr{C}^{\alpha \eta_{1}, \alpha \eta_{2}+\beta}\left(\omega^{1 / \alpha}\right)$ generates the following $\left(g_{\alpha \eta_{1}}, g_{\alpha \eta_{2}+\beta}\right)$-regularized resolvent family:

$$
\begin{array}{r}
S_{\alpha \eta_{1}, \alpha \eta_{2}+\beta}(t) x:=\int_{0}^{\infty} \psi_{\alpha, \beta}(t, s) S_{\eta_{1}, \eta_{2}}(s) x d s, \\
t>0, \quad x \in X,
\end{array}
$$

for $0<\alpha<1$ and $\beta \geq 0$. Moreover the following equality holds:

$$
S_{\alpha \eta_{1}, \alpha \eta_{2}+\beta}(t) x=\left(g_{\beta} * S_{\alpha \eta_{1}, \alpha \eta_{2}}\right)(t) x, \quad t>0, x \in X,
$$

for $\beta>0$.

Proof. First we show that $\left\{S_{\alpha \eta_{1}, \alpha \eta_{2}+\beta}(t)\right\}_{t>0}$ is Laplace transformable of parameter $\omega^{1 / \alpha}$. Note that using Theorem 3(ii) we get that

$$
\begin{aligned}
& \int_{0}^{\infty} e^{-\lambda t} S_{\alpha \eta_{1}, \alpha \eta_{2}+\beta}(t) x d t \\
& \quad=\int_{0}^{\infty}\left(\int_{0}^{\infty} e^{-\lambda t} \psi_{\alpha, \beta}(t, s) d t\right) S_{\eta_{1}, \eta_{2}}(s) x d s \\
& =\lambda^{-\beta} \int_{0}^{\infty} e^{-\lambda^{\alpha s}} S_{\eta_{1}, \eta_{2}}(s) x d s \\
& =\lambda^{\alpha \eta_{1}-\left(\alpha \eta_{2}+\beta\right)}\left(\lambda^{\alpha \eta_{1}}-A\right)^{-1} x,
\end{aligned}
$$

for $\lambda>\omega^{1 / \alpha}$, and $\left(\omega^{\eta_{1}}, \infty\right)=\left(\left(\omega^{1 / \alpha}\right)^{\alpha \eta_{1}}, \infty\right) \subset \rho(A)$. 
The family $\left\{S_{\alpha \eta_{1}, \alpha \eta_{2}+\beta}(t)\right\}_{t>0}$ is strongly continuous on $(0, \infty)$ and now we prove the strong continuity at the origin. Let $x \in X$, and then

$$
\begin{aligned}
& \frac{\left\|S_{\alpha \eta_{1}, \alpha \eta_{2}+\beta}(t) x-g_{\alpha \eta_{2}+\beta}(t) x\right\|}{g_{\alpha \eta_{2}+\beta}(t)} \\
& \leq \int_{0}^{\infty} \frac{\psi_{\alpha, \beta}(t, s)}{g_{\alpha \eta_{2}+\beta}(t)}\left\|S_{\eta_{1}, \eta_{2}}(s) x-g_{\eta_{2}}(s) x\right\| d s \\
& =\Gamma\left(\alpha \eta_{2}+\beta\right) \int_{0}^{\infty} t^{\alpha-\alpha \eta_{2}} W_{-\alpha, \beta}(-u) \\
& \cdot\left\|S_{\eta_{1}, \eta_{2}}\left(u t^{\alpha}\right) x-g_{\eta_{2}}\left(u t^{\alpha}\right) x\right\| d u \\
& =\Gamma\left(\alpha \eta_{2}+\beta\right) \int_{0}^{\infty} \frac{g_{\eta_{2}}(u) W_{-\alpha, \beta}(-u)}{g_{\eta_{2}}\left(u t^{\alpha}\right)} \\
& \cdot\left\|S_{\eta_{1}, \eta_{2}}\left(u t^{\alpha}\right) x-g_{\eta_{2}}\left(u t^{\alpha}\right) x\right\| d u,
\end{aligned}
$$

where we have used Theorem 3(vi). We apply the dominated convergence theorem to the above term and Proposition 1(i) to conclude that

$$
\frac{\left\|S_{\alpha \eta_{1}, \alpha \eta_{2}+\beta}(t) x-g_{\alpha \eta_{2}+\beta}(t) x\right\|}{g_{\alpha \eta_{2}+\beta}(t)} \longrightarrow 0, \quad t \longrightarrow 0^{+},
$$

since $S_{\eta_{1}, \eta_{2}}(t)$ is a $\left(g_{\eta_{1}}, g_{\eta_{2}}\right)$-regularized resolvent family. Finally, by Theorem 10 , we obtain that the family $\left\{S_{\alpha \eta_{1}, \alpha \eta_{2}+\beta}(t)\right\}_{t>0}$ is a $\left(g_{\alpha \eta_{1}}, g_{\alpha \eta_{2}+\beta}\right)$-regularized resolvent family generated by $A$. The proof of equality (52) is a straightforward consequence of Theorem 3(v).

Remark 13. Note that the above subordination theorem extends some subordination results which have been considered in this paper and previous papers.

(i) Now, we consider the family of functions $\left(\psi_{\gamma, \delta-\gamma}(t,(\cdot))\right)_{t>0}$ which is a $\left(g_{\gamma}, g_{\delta}\right)$-regularized resolvent family for $0<\gamma<1$ and $\delta \geq \gamma$ (see Example 11). Then we apply Theorem 12 for $0<\alpha<1$ and $\beta>0$, and we obtain the formula

$$
\psi_{\alpha \gamma, \beta+\alpha(\delta-\gamma)}(t,(\cdot))=\int_{0}^{\infty} \psi_{\alpha, \beta}(t, s) \psi_{\gamma, \delta-\gamma}(s,(\cdot)) d s, \quad t>0,
$$

which is shown in Theorem 8 .

(ii) If $0<\eta_{1} \leq 2$ and $\eta_{2}=1$ in Theorem 12 , we retrieve the subordination principle for $\left(g_{\alpha \eta_{1}}, 1\right)$-regularized resolvent families given in [9, Theorem 3.1] and [10, Theorem 3.1] for $0<\alpha<1$ and $\beta=1-\alpha$.

(iii) By [12, Theorem 2.8(i)], given a $\left(g_{\eta_{1}}, g_{\eta_{2}}\right)$-regularized resolvent family generated by $A$, we obtain a $\left(g_{\gamma_{1}}, g_{\gamma_{2}}\right)$ regularized resolvent family generated by $A$, where $0<\gamma_{1}<\eta_{1}$ and $\gamma_{2}=1-\gamma_{1} / \eta_{1}+\left(\gamma_{1} / \eta_{1}\right) \eta_{2}$. This is a particular case of Theorem 12 for $\alpha=\gamma_{1} / \eta_{1}$ and $\beta=1-\gamma_{1} / \eta_{1}$.
Corollary 14. Let $0<\eta \leq 2$ and $0<\alpha<1$. If $\left\{S_{\eta, \eta}(t)\right\}_{t>0}$ is a $\left(g_{\eta}, g_{\eta}\right)$-regularized resolvent family generated by $A \in \mathscr{C}^{\eta, \eta}(\omega)$, then $A \in \mathscr{C}^{\alpha \eta, \alpha \eta}\left(\omega^{1 / \alpha}\right)$ generates the following $\left(g_{\alpha \eta}, g_{\alpha \eta}\right)$ regularized resolvent family:

$$
S_{\alpha \eta, \alpha \eta}(t) x:=\int_{0}^{\infty} \psi_{\alpha, 0}(t, s) S_{\eta, \eta}(s) x d s, \quad t>0, x \in X .
$$

In particular, we have the following remarkable particular cases.

(i) If $A$ generates a $C_{0}$-semigroup $\{T(t)\}_{t>0}$, that is, a $\left(g_{1}\right.$, $\left.g_{1}\right)$-regularized resolvent family, satisfying $\|T(t)\| \leq$ $M e^{\omega t}, t \geq 0$, then $A \in \mathscr{C}^{\alpha, \alpha}\left(\omega^{1 / \alpha}\right)$ generates the following $\left(g_{\alpha}, g_{\alpha}\right)$-regularized resolvent family:

$S_{\alpha, \alpha}(t) x:=\int_{0}^{\infty} \psi_{\alpha, 0}(t, s) T(s) x d s, \quad t>0, x \in X$.

(ii) Let $\beta \in(1,2]$ and $\left\{S_{\beta, \beta}(t)\right\}_{t>0}$ be a $\left(g_{\beta}, g_{\beta}\right)$-regularized resolvent family generated by $A \in \mathscr{C}^{\beta, \beta}(\omega)$. Then $A \in$ $\mathscr{C}^{1,1}\left(\omega^{\beta}\right)$ generates the following $C_{0}$-semigroup:

$T(t) x:=\int_{0}^{\infty} \psi_{1 / \beta, 0}(t, s) S_{\beta, \beta}(s) x d s, \quad t>0, x \in X$.

In the case that $A$ is the generator of an exponentially bounded sine function $\|S(t)\| \leq M e^{\omega t},(t \geq 0)$ (see details in [5, Section 3.15]), that is, $(S(t))_{t>0}$ is a $\left(g_{2}\right.$, $g_{2}$ )-regularized resolvent family, then $A$ generates the following $\mathrm{C}_{0}$-semigroup:

$$
T(t) x:=\frac{1}{2 \sqrt{\pi}} t^{-3 / 2} \int_{0}^{\infty} s e^{-s^{2} / 4 t} S(s) x d s, \quad t>0, x \in X,
$$

with exponential bound $\omega^{2}$, which is proved in [24, Theorem 5.2], and see also [25, Proposition 2.5 and Theorem 2.6].

\section{Applications, Examples, and Final Comments}

In this last section we present some applications of our results to fractional Cauchy problems and classical semigroups in Lebesgue spaces. Now we recall some basic definitions on fractional Cauchy problems. Let $f \in C_{c}^{(\infty)}\left(\mathbb{R}_{+} ; X\right)$; we call Riemann-Liouville fractional integral of $f$ of order $\gamma>0$, $I^{-\gamma} f$ to the function given by

$$
I^{-\gamma} f(t):=g_{\gamma} * f(t)=\int_{0}^{t} \frac{(t-s)^{\gamma-1}}{\Gamma(\gamma)} f(s) d s, \quad t \geq 0,
$$

and the Riemann-Liouville fractional derivative of $f$ of order $\gamma>0$ is given by

$$
{ }_{R} D_{t}^{\gamma} f(t):=\frac{d^{n}}{d t^{n}}\left(I^{-(n-\gamma)} f\right)(t), \quad t \geq 0,
$$


and with $n=[\gamma]+1$. Also, we consider the Caputo fractional derivative of $f$ of order $\gamma>0$,

$$
{ }_{C} D_{t}^{\gamma} f(t):=g_{n-\gamma} * f^{(n)}(t)=I^{-(n-\gamma)} f^{(n)}(t), \quad t \geq 0,
$$

with $n=[\gamma]+1$; see, for example [14, 15] and [11, Section 4]. Note that, in the above definitions, the function $f$ can be taken in a larger space than $C_{c}^{(\infty)}\left(\mathbb{R}_{+}\right)$where the definitions make sense.

5.1. Fractional Powers in Fractional Cauchy Problems. The next results extend [11, Theorem 4.9(a), (c)]: for $0<\alpha<$ 1 , the solutions of Caputo fractional problems are $\left(g_{\alpha}, 1\right)$ regularized resolvent families (see [10, Definition 2.3]) which may be obtained by integration from $\left(g_{\alpha}, g_{\alpha}\right)$-regularized resolvent families by (52). Note that these are solutions of Riemann-Liouville fractional problems; see [17, Theorem 1.1].

Theorem 15. Let $\alpha, \gamma \in(0,1)$ and $A$ be the generator of $a$ uniformly bounded $C_{0}$-semigroup $\{T(t)\}_{t>0} \subset \mathscr{B}(X)$.

(i) The fractional Cauchy problem

$$
\begin{aligned}
& { }_{R} D_{t}^{\alpha} v(t)=A v(t), \quad t>0, \\
& \left(g_{1-\alpha} * v\right)(0)=x \in D(A)
\end{aligned}
$$

is well-posed and its unique solution is given by

$$
v(t)=\int_{0}^{\infty} \psi_{\alpha, 0}(t, s) T(s) x d s, \quad t>0 .
$$

(ii) The fractional Cauchy problem

$$
\begin{gathered}
{ }_{R} D_{t}^{\gamma} v(t)=-(-A)^{\alpha} v(t), \quad t>0, \\
\left(g_{1-\gamma} * v\right)(0)=x \in D(A)
\end{gathered}
$$

is well-posed and its unique solution is given by

$$
v(t)=\int_{0}^{\infty} \Psi_{\gamma, \alpha}(t, s) T(s) x d s, \quad t>0 .
$$

Proof. (i) By Corollary 14(i), the operator $A$ generates a $\left(g_{\alpha}, g_{\alpha}\right)$-regularized resolvent family which provides the solution of fractional Cauchy problem (64); see [17, Theorem 1.1]. (ii) By [4, Chapter IX], the operator $-(-A)^{\alpha}$ generates a $C_{0}$-semigroup $\left\{T^{(\alpha)}(t)\right\}_{t>0}$ given by (5). By part (i), Fubini theorem, and the definition of $\Psi_{\gamma, \alpha}$ in Corollary 4 , we conclude the proof.

5.2. Convolution Semigroups on $L^{p}\left(\mathbb{R}^{n}\right)$. Let $\{T(t)\}_{t \geq 0}$ be a uniformly bounded convolution semigroup in $\mathbb{R}^{n}$ generated by $A$; that is,

$$
\begin{array}{r}
T(t) f(x)=\left(k_{t} * f\right)(x)=\int_{\mathbb{R}^{n}} k_{t}(x-y) f(y) d y, \\
t>0, \quad x \in \mathbb{R}^{n} .
\end{array}
$$

Two well-known examples of convolution semigroups are Gaussian and Poisson semigroups, $g_{t}(x)=(1 /$ $\left.(4 \pi t)^{n / 2}\right) e^{-|x|^{2} / 4 t}$ and $p_{t}(x)=\left(\Gamma((n+1) / 2) / \pi^{(n+1) / 2}\right)\left(t /\left(t^{2}+\right.\right.$ $\left.\left.|x|^{2}\right)^{(n+1) / 2}\right)$, whose generators are the Laplacian $\Delta$ and $-(-\Delta)^{1 / 2}$, respectively; see, for example, [4, Chapter IX]. Then, by Theorem 15(i), the solution of the RiemannLiouville fractional diffusion problem of order $0<\alpha<1$

$$
\begin{gathered}
{ }_{R} D_{t}^{\alpha} u(t, x)=A u(t, x), \quad t>0, \\
\left(g_{1-\alpha} * u(\cdot, x)\right)(0)=f(x)
\end{gathered}
$$

is given by

$$
u(t, x)=\int_{\mathbb{R}^{n}}\left(\int_{0}^{\infty} \psi_{\alpha, 0}(t, s) k_{s}(x-y) d s\right) f(y) d y .
$$

In addition, to the particular case of the Laplacian, we obtain the solution of the Caputo fractional diffusion problem of order $0<\alpha<1$,

$$
\begin{gathered}
{ }_{C} D_{t}^{\alpha} v(t, x)=\Delta v(t, x), \quad t>0, \\
v(0, x)=f(x),
\end{gathered}
$$

(considered in [11, Example 4.13]) which is given by $v(t, x)=$ $\int_{0}^{t} g_{1-\alpha}(t-s) u(s, x) d s$, for $t>0$.

5.3. Multiplication Families on $C_{0}\left(\mathbb{R}^{n}\right)$. Let $\{T(t)\}_{t \geq 0}$ be a multiplication semigroup in $\mathbb{R}^{n}$ generated by $q(x)$; that is,

$$
T(t) f(x)=e^{t q(x)} f(x), \quad x \in \mathbb{R}^{n}, t>0 .
$$

Some examples are $q(x)=-4 \pi^{2}|x|^{2},-2 \pi|x|,-\log (1+$ $\left.4 \pi^{2}|x|^{2}\right)$, treated in [26]. Then, by Theorem 15(i), the solution of the Riemann-Liouville fractional diffusion problem of order $0<\alpha<1$

$$
\begin{array}{cc}
{ }_{R} D_{t}^{\alpha} u(t, x)=q(x) u(t, x), & t>0, \\
\left(g_{1-\alpha} * u(\cdot, x)\right)(0)=f(x), & x \in \mathbb{R}^{n},
\end{array}
$$

is given by

$$
\begin{aligned}
u(t, x) & =\int_{0}^{\infty} \psi_{\alpha, 0}(t, s) e^{s q(x)} f(x) d s \\
& =t^{\alpha-1} E_{\alpha, \alpha}\left(q(x) t^{\alpha}\right) f(x), \quad t>0, \quad x \in \mathbb{R}^{n},
\end{aligned}
$$

where we have applied Theorem 3(iii). Even more, by Theorem 15(ii), the solution of the Riemann-Liouville fractional diffusion problem of order $0<\alpha, \gamma<1$,

$$
\begin{gathered}
{ }_{R} D_{t}^{\gamma} u(t, x)=-(-q(x))^{\alpha} u(t, x), \quad t>0, \\
\left(g_{1-\gamma} * u(\cdot, x)\right)(0)=f(x)
\end{gathered}
$$

is given by

$$
\begin{aligned}
u(t, x) & =\int_{0}^{\infty} \Psi_{\gamma, \alpha}(t, s) e^{s q(x)} f(x) d s \\
& =t^{\gamma-1} E_{\gamma, \gamma}\left(-(-q(x))^{\alpha} t^{\gamma}\right) f(x), \quad t>0,
\end{aligned}
$$

where we have applied Theorem 3(ii) and (iii). 


\section{Conflict of Interests}

The authors declare that there is no conflict of interests regarding the publication of this paper.

\section{Acknowledgments}

The authors thank Professor Carlos Lizama for some comments and nice ideas that have contributed to improve the final version of this paper. The authors also thank the referee for several comments and, in particular, [8]. Authors have been partially supported by Project MTM2013-42105-P, DGIFEDER, of the MCYTS, Project E-64, D. G. Aragón, and Project UZCUD2014-CIE-09, Universidad de Zaragoza.

\section{References}

[1] R. Schilling, R. Song, and Z. Vondracek, Bernstein Functions, vol. 37 of De Gruyter Studies in Mathematics, Walter de Gruyter, Berlin, Germany, 2010.

[2] S. Bochner, "Diffusion equation and stochastic processes," Proceedings of the National Academy of Sciences of the United States of America, vol. 35, pp. 368-370, 1949.

[3] S. Bochner, Harmonic Analysis and the Theory of Probability, California Monographs in Mathematical Sciences, University of California Press, Berkeley, Calif, USA, 1955.

[4] K. Yosida, Functional Analysis, vol. 123 of A Series of Comprehensive Studies in Mathematics, Springer, Berlin, Germany, 5th edition, 1978.

[5] W. Arendt, C. J. K. Batty, M. Hieber, and F. Neubrander, VectorValued Laplace Transforms and Cauchy Problems, vol. 96 of Monographs in Mathematics, Birkhäuser, 2nd edition, 2011.

[6] C. Lizama, "Regularized solutions for abstract Volterra equations," Journal of Mathematical Analysis and Applications, vol. 243, no. 2, pp. 278-292, 2000.

[7] J. Präuss, Evolutionary Integral Equations and Applications, vol. 87 of Monographs in Mathematics, Birkhäauser, Basel, Switzerland, 1993.

[8] C. Lizama and H. Prado, "On duality and spectral properties of (a, k)-regularized resolvents," Proceedings of the Royal Society of Edinburgh, Section A: Mathematics, vol. 139, no. 3, pp. 505-517, 2009.

[9] E. G. Bazhlekova, "Subordination principle for fractional evolution equations," Fractional Calculus and Applied Analysis, vol. 3, no. 3, pp. 213-230, 2000.

[10] E. G. Bajlekova, Fractional evolution equations in Banach spaces [Thesis], University Press Facilities, Eindhoven University of Technology, 2001.

[11] M. Li, C. Chen, and F.-B. Li, "On fractional powers of generators of fractional resolvent families," Journal of Functional Analysis, vol. 259, no. 10, pp. 2702-2726, 2010.

[12] M. Kostic, “ $(a ; k)$-regularized $C$-resolvent families: regularity and local properties," Abstract and Applied Analysis, vol. 2009, Article ID 858242, 27 pages, 2009.

[13] J. Peng and K. Li, "A note on property of the Mittag-Leffler function," Journal of Mathematical Analysis and Applications, vol. 370, no. 2, pp. 635-638, 2010.

[14] F. Mainardi, Fractional Calculus and Waves in Linear Viscoelasticity: An Introduction to Mathematical Models, Imperial College Press, London, UK, 2010.
[15] K. S. Miller and B. Ross, An Introduction to the Fractional Calculus and Fractional Differential Equations, John Wiley \& Sons, New York, NY, USA, 1993.

[16] Y.-N. Li and H.-R. Sun, "Integrated fractional resolvent operator function and fractional abstract Cauchy problem," Abstract and Applied Analysis, vol. 2014, Article ID 430418, 9 pages, 2014.

[17] Z.-D. Mei, J.-G. Peng, and Y. Zhang, "A characteristic of fractional resolvents," Fractional Calculus and Applied Analysis, vol. 16, no. 4, pp. 777-790, 2013.

[18] E. M. Wright, "On the coefficients of power series having exponential singularities," Journal of the London Mathematical Society, vol. 8, pp. 71-79, 1933.

[19] F. Mainardi, G. Pagnini, and R. Gorenflo, "Mellin transform and subordination laws in fractional diffusion processes," Fractional Calculus \& Applied Analysis, vol. 6, no. 4, pp. 441-459, 2003.

[20] F. Mainardi, Y. Luchko, and G. Pagnini, "The fundamental solution of the space-time fractional diffusion equation," Fractional Calculus and Applied Analysis, vol. 4, no. 2, pp. 153-192, 2001.

[21] P. J. Miana, "Integrated groups and smooth distribution groups," Acta Mathematica Sinica, vol. 23, no. 1, pp. 57-64, 2007.

[22] V. A. Ditkine and A. Proudnikov, Calcul Opérationnel, Mir Moscou, 2nd edition, 1983.

[23] C. Chen and M. Li, "On fractional resolvent operator functions," Semigroup Forum, vol. 80, no. 1, pp. 121-142, 2010.

[24] W. Arendt and H. Kellermann, "Integrated solutions of Volterra integrodifferential equations and applications," in Volterra Integrodifferential Equations in Banach Spaces and Applications, vol. 190 of Pitman Research Notes in Mathematics Series, pp. 21-51, Longman, New York, NY, USA, 1987.

[25] V. Keyantuo, "Integrated semigroups and related partial differential equations," Journal of Mathematical Analysis and Applications, vol. 212, no. 1, pp. 135-153, 1997.

[26] J. S. Campos-Orozco and J. E. Galé, "Special functions as subordinated semigroups on the real line," Semigroup Forum, vol. 84, no. 2, pp. 284-300, 2012. 


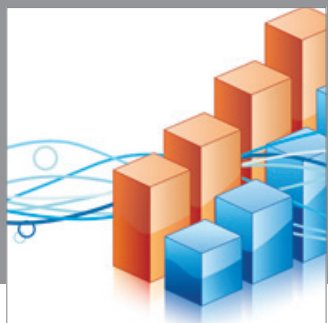

Advances in

Operations Research

mansans

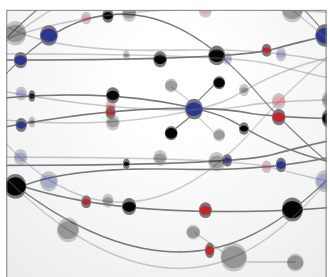

The Scientific World Journal
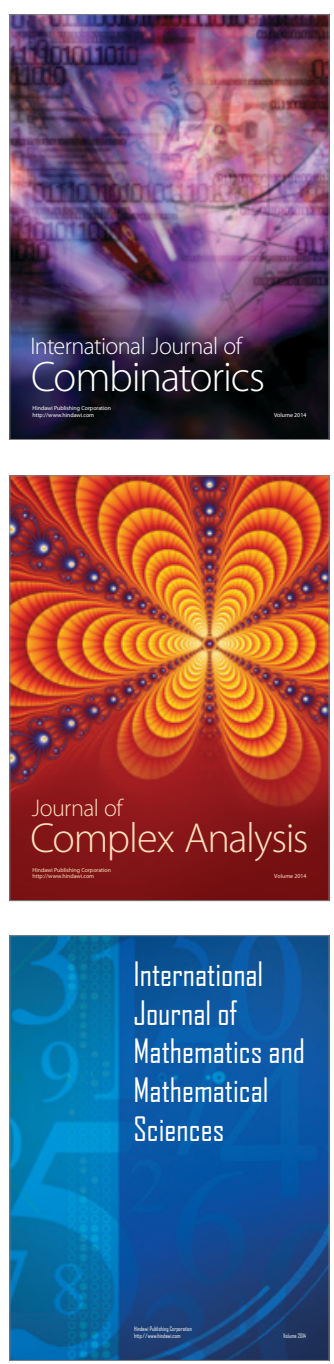
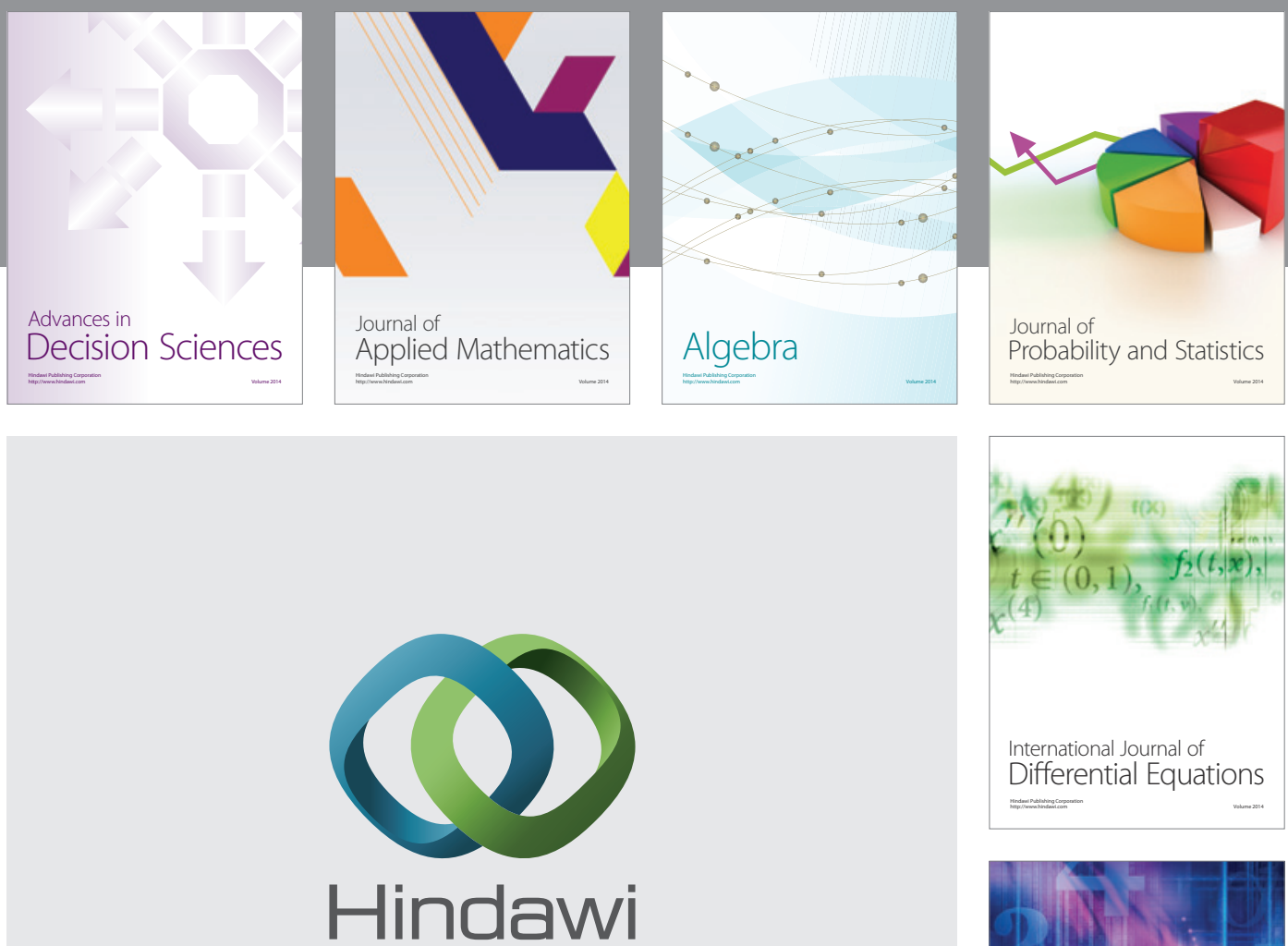

Submit your manuscripts at http://www.hindawi.com
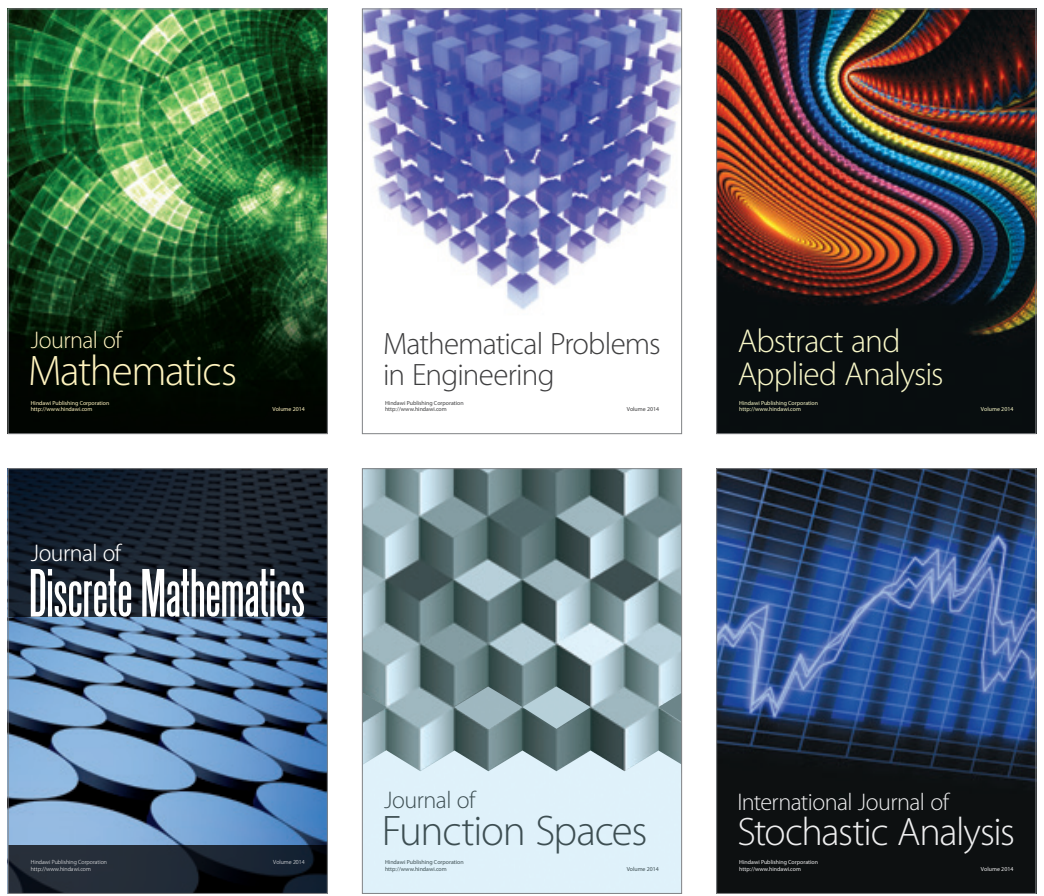

Journal of

Function Spaces

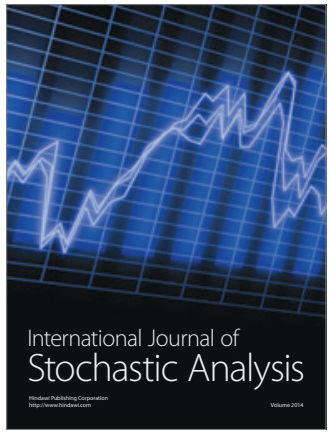

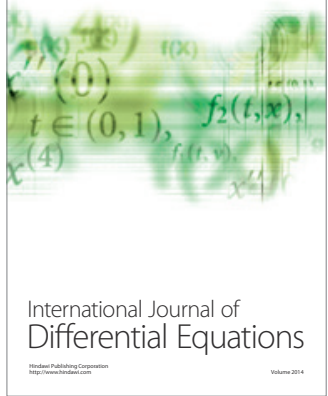
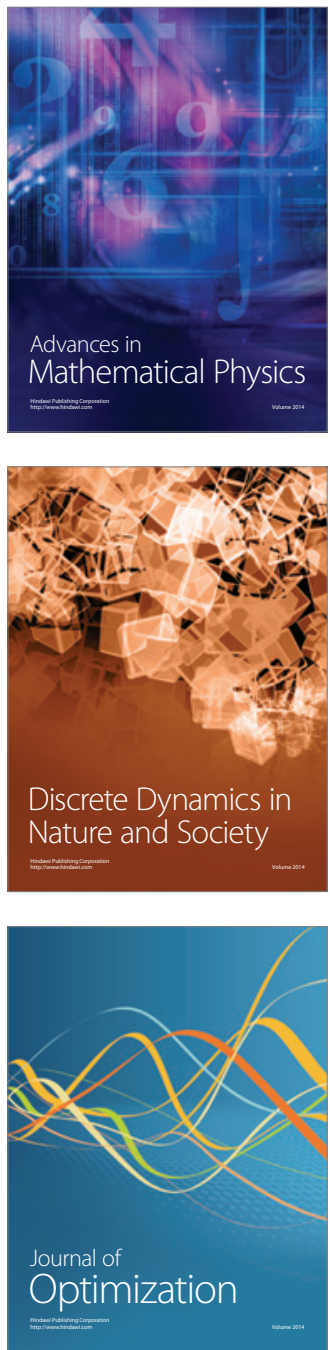